\title{
Knot Theory from the Perspective of Field and String Theory
}

\author{
Jose M. F. Labastida* \\ Departamento de Física de Partículas \\ Universidade de Santiago de Compostela \\ E-15706 Santiago de Compostela, SPAIN \\ E-mail: 'Labasti@fpaxpínsc.esi
}

ABSTRACT: I present a summary of the recent progress made in field and string theory which has led to a reformulation of quantum-group polynomial invariants for knots and links into new polynomial invariants whose coefficients can be described in topological terms. The approach opens a new point of view in the theory of knot and link invariants.

During the last years the theory of knot and link invariants has experienced important progress. The confluence of Chern-Simons gauge theory and string theory has led to a very powerful new approach which provides a topological interpretation for the integer coefficients of a reformulated version of quantum-group polynomial invariants. The main goal of this short note is to present a summary of these recent developments.

Chern-Simons gauge theory is a topological quantum field theory whose action is built out of a Chern-Simons term involving as gauge field a gauge connection associated to a group $G$ on a three-manifold $M$. Its natural observables are Wilson loops, $W_{R}^{K}$, where $K$ is a loop and $R$ a representation of the gauge group. The vacuum expectation values of products of these operators are topological invariants which are related to quantum-group polynomial invariants. Given a link $\mathcal{L}$ of $L$ components, $K_{1}, K_{2}, \ldots, K_{L}$, one computes correlators of the form $\left\langle W_{R_{1}}^{K_{1}} \cdots W_{R_{L}}^{K_{L}}\right\rangle$, where $R_{1}, R_{2}, \ldots, R_{L}$ are representations associated to each component. For $S U(N)$ as gauge group these quantities turn out to be polynomials in $q=\mathrm{e}^{\frac{2 \pi i}{k+N}}$ and $\lambda=q^{N}$ with integer coefficients, being $k$ the Chern-Simons integer parameter.

Witten found out in 1988 [i], using non-perturbative methods, that these correlators lead to quantum-group polynomial invariants for knots and links [2] have been also developed for Chern-Simons gauge theory, lading to important connections related to Vassiliev invariants. A summary of these developments can be found in a recent review [3]. In this short note I will concentrate in the new perspective emerged after

\footnotetext{
${ }^{*}$ Speaker.
} 
studying the large $N$ expansion of Chern-Simons gauge theory. The discussion will be restricted to the case of knots on $S^{3}$ with gauge group $S U(N)$.

Besides the perturbative expansion, gauge theories with gauge group $S U(N)$ admit a large- $N$ expansion. In this expansion correlators are expanded in powers of $1 / N$ while keeping the 't Hooft coupling $t=N x$ fixed, being $x$ the coupling constant of the gauge theory. For example, for the free energy of the theory one has the general form,

$$
F=\sum_{\substack{g \geq 0 \\ h \geq 1}}^{\infty} C_{g, h} N^{2-2 g} t^{2 g-2+h} .
$$

In the case of Chern-Simons gauge theory, the coupling constant is $x=\frac{2 \pi i}{k+N}$ after taking into account the standard shift in $k$. This large- $N$ expansion resembles a string theory expansion and indeed the quantities $C_{g, h}$ can be identified with the partition function of a topological open string with $g$ handles and $h$ boundaries, with $N$ D-branes on $S^{3}$ in an ambient six-dimensional target space $T^{*} S^{3}$. This was pointed out by Witten in 1992 [4] The result makes a connection between a topological three-dimensional field theory and topological string theory.

An important breakthrough took place in 1998 after the discovery of a new approach

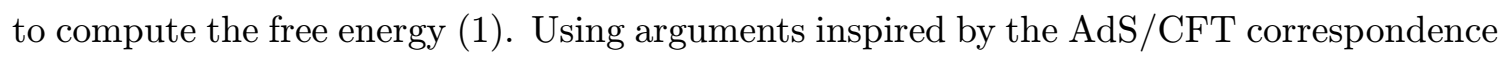
(see [i5] for a review), Gopakumar and Vafa [6] provided a closed-string theory interpretation of the free energy (1) They conjectured that it can be expressed as $F=\sum_{g \geq 0}^{\infty} N^{2-2 g} F_{g}(t)$, where $F_{g}(t)$ corresponds to the partition function of a topological closed string theory on the non-compact Calabi-Yau manifold $X$ called the resolved conifold, $\mathcal{O}(-1) \oplus \mathcal{O}(-1) \rightarrow \mathbf{P}^{1}$, being $t$ the flux of the $B$-field through $\mathbf{P}^{1}$. The quantities $F_{g}(t)$ have been computed using physical [i6] and mathematical arguments [i]i], proving the conjecture.

The study of observables in this new context was first faced by Ooguri and Vafa, and

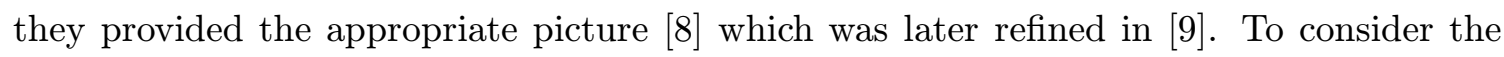
presence of Wilson loops it is convenient to introduce a particular generating functional. First, one performs a change of basis from representations $R$ to conjugacy classes $C(\vec{k})$ of the symmetric group, labeled by vectors $\vec{k}=\left(k_{1}, k_{2}, \ldots\right)$ with $k_{i} \geq 0$, and $|\vec{k}|=\sum_{j} k_{j}>0$. The change of basis is $W_{\vec{k}}=\sum_{R} \chi_{R}(C(\vec{k})) W_{R}$, where $\chi_{R}$ are characters of the permutation group $S_{\ell}$ of $\ell=\sum_{j} j k_{j}$ elements ( $\ell$ is also the number of boxes of the Young tableau associated to $R$ ). Second, one introduces the generating functional, $F(V)=\log Z(V)=$ $\sum_{\vec{k}} \frac{|C(\vec{k})|}{\ell !} W_{\vec{k}}^{(c)} \Upsilon_{\vec{k}}(V)$, where $Z(V)=\sum_{\vec{k}} \frac{|C(\vec{k})|}{\ell !} W_{\vec{k}} \Upsilon_{\vec{k}}(V)$ and $\Upsilon_{\vec{k}}(V)=\prod_{j}\left(\operatorname{Tr} V^{j}\right)^{k_{j}}$. In these expressions $|C(\vec{k})|$ denotes the number of elements of the class $C(\vec{k})$ in $S_{\ell}$. The reason behind the introduction of this generating functional is that the large- $N$ structure of the connected Wilson loops, $W_{\vec{k}}^{(c)}$, turns out to be very simple:

$$
\frac{|C(\vec{k})|}{\ell !} W_{\vec{k}}^{(c)}=\sum_{g=0}^{\infty} x^{2 g-2+|\vec{k}|} F_{g, \vec{k}}(\lambda),
$$

where $\lambda=\mathrm{e}^{t}$ and $t=N x$ is the 't Hooft coupling. Writing $x=t / N$, it corresponds to a power series expansion in $1 / N$. As before, the expansion looks like a perturbative 
series in string theory where $g$ is the genus and $|\vec{k}|$ is the number of holes. Ooguri and

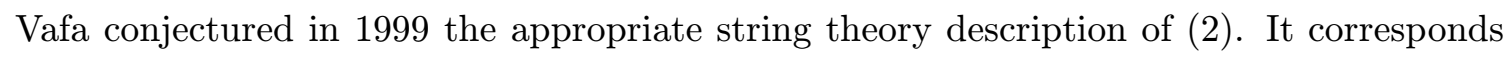
to an open topological string theory whose target space is the resolved conifold $X$. The contribution from this theory leads to open-string analogs of Gromov-Witten invariants.

To describe in detail the fact that one is dealing with open strings, some new data needs to be introduced. Here is where the knot intrinsic to the Wilson loop enters. Given a knot $K$ on $S^{3}$, let us associate to it a Lagrangian submanifold $C_{K}$ with $b_{1}=1$ in the resolved conifold $X$ and consider a topological open string on it. The contributions in this open topological string are localized on holomorphic maps $f: \Sigma_{g, h} \rightarrow X$ with $h=|\vec{k}|$ which satisfy: $f_{*}\left[\Sigma_{g, h}\right]=\mathcal{Q}$, and $f_{*}[C]=j[\gamma]$ for $k_{j}$ oriented circles $C$. In these expressions $\gamma \in H_{1}\left(C_{K}, \mathbf{Z}\right)$, and $\mathcal{Q} \in H_{2}\left(X, C_{K}, \mathbf{Z}\right)$, i.e., the map is such that $k_{j}$ boundaries of $\Sigma_{g, h}$ wrap the knot $j$ times, and $\Sigma_{g, h}$ itself gets mapped to a relative two-homology class characterized by the Lagrangian submanifold $C_{K}$. The number of these maps (defined in an appropriate form) constitutes the open-string analog of Gromov-Witten invariants. They will be denoted by $N_{g, \vec{k}}^{\mathcal{Q}}$. The quantity $F_{g, \vec{k}}(\lambda)$ in $(\underset{2}{2})$ takes the form:

$$
F_{g, \vec{k}}(\lambda)=\sum_{\mathcal{Q}} N_{g, \vec{k}}^{\mathcal{Q}} \mathrm{e}^{\int_{\mathcal{Q}} \omega}, \quad t=\int_{\mathbf{P}^{1}} \omega
$$

where $\omega$ is the Kähler class of the Calabi-Yau manifold $X$ and $\lambda=\mathrm{e}^{t}$. For any $\mathcal{Q}$, one can always write $\int_{\mathcal{Q}} \omega=Q t$ where $Q$ is in general a half-integer number. Therefore, $F_{g, \vec{k}}(\lambda)$ is a polynomial in $\lambda^{ \pm \frac{1}{2}}$ with rational coefficients.

The result $\left(\begin{array}{l}\overline{3} \\ 3\end{array}\right)$ constitutes a first step to provide a representation where one can assign a geometrical interpretation to the integer coefficients of the quantum-group invariants. Notice that to match a polynomial invariant to $\left(\begin{array}{l}(\overline{3} \\ 2\end{array}\right)$, after obtaining its connected part, one must expand it in $x$ after setting $q=\mathrm{e}^{x}$ keeping $\lambda$ fixed. One would like to have a refined

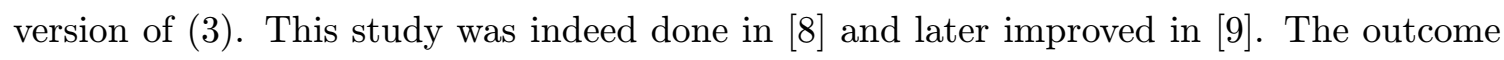
is that $F(V)$ can be expressed in terms of integer invariants related to topological strings.

To present these results one needs to perform first a reformulation of the quantumgroup invariants or vacuum expectation values of Wilson loops. Instead of considering $W_{R}(q, \lambda)$, a corrected version of it, $f_{R}(q, \lambda)$, will be studied. These reformulated polynomial invariants have the form:

$$
\begin{aligned}
f_{R}(q, \lambda)= & \sum_{d, m=1}^{\infty}(-1)^{m-1} \frac{\mu(d)}{d m} \sum_{\left\{\vec{k}^{(j)}, R_{j}\right\}} \chi_{R}\left(C\left(\left(\sum_{j=1}^{m} \vec{k}^{(j)}\right)_{d}\right)\right) \\
& \times \prod_{j=1}^{m} \frac{\left|C\left(\vec{k}^{(j)}\right)\right|}{\ell_{j} !} \chi_{R_{j}}\left(C\left(\vec{k}^{(j)}\right)\right) W_{R_{j}}\left(q^{d}, \lambda^{d}\right),
\end{aligned}
$$

where $\left(\vec{k}_{d}\right)_{d i}=k_{i}$ and zero otherwise. In this expression $\mu(d)$ is the Moebius function. The reformulated polynomial invariants $f_{R}(q, \lambda)$ are just quantum-group invariants $W_{R}(q, \lambda)$ plus lower order terms, understanding by this terms which contain quantum-group invariants carrying representations whose associated Young tableaux have a lower number of 
boxes. For example, for the simplest cases:

$$
\begin{aligned}
& f_{\square}(q, \lambda)=W_{\square}(q, \lambda), \\
& f_{\text {口 }}(q, \lambda)=W_{\text {口 }}(q, \lambda)-\frac{1}{2}\left(W_{\square}(q, \lambda)^{2}+W_{\square}\left(q^{2}, \lambda^{2}\right)\right) \text {, } \\
& f_{\text {日 }}(q, \lambda)=W_{\text {日 }}(q, \lambda)-\frac{1}{2}\left(W_{\square}(q, \lambda)^{2}-W_{\square}\left(q^{2}, \lambda^{2}\right)\right) .
\end{aligned}
$$

The nice feature of the reformulated quantities is that $F(V)$ acquires a very simple form in terms of them:

$$
F(V)=\sum_{d=1}^{\infty} \sum_{R} \frac{1}{d} f_{R}\left(q^{d}, \lambda^{d}\right) \operatorname{Tr} V^{d},
$$

and therefore they seem to be the right quantities to express an alternative point of view in which the embedded Riemann surfaces can be regarded as D-branes.

Two more ingredients are needed to present the conjectured form of the reformulated $f_{R}(q, \lambda)$. One needs the Clebsch-Gordon coefficients $C_{R, R^{\prime}, R^{\prime \prime}}$ of the symmetric group (they satisfy $V_{R} \otimes V_{R^{\prime}}=\sum_{R^{\prime \prime}} C_{R, R^{\prime}, R^{\prime \prime}} V_{R^{\prime \prime}}$ ), and monomials $S_{R}(q)$ defined as follows: $S_{R}(q)=(-1)^{d} q^{d-\frac{\ell-1}{2}}$ if $R$ is a hook representation, with $\ell-d$ boxes in the first row, and $S_{R}(q)=0$ otherwise. The conjecture presented in $[9]$ states that the reformulated invariants have the form:

$$
f_{R}(q, \lambda)=\sum_{g \geq 0} \sum_{Q, R^{\prime}, R^{\prime \prime}} C_{R R^{\prime} R^{\prime \prime}} N_{R^{\prime}, g, Q} S_{R^{\prime \prime}}(q)\left(q^{\frac{1}{2}}-q^{-\frac{1}{2}}\right)^{2 g-1} \lambda^{Q},
$$

where $N_{R, g, Q}$ are integer invariants which posses a geometric interpretation. These quantities are alternative integers in Gromov-Witten theory. They can be described in terms of the moduli space of Riemann surfaces with boundaries embedded into a Calabi-Yau manifold. Their geometrical interpretation has been treated recently in [i]

The structure present in (iii) has been verified for a variety of non-trivial knots and

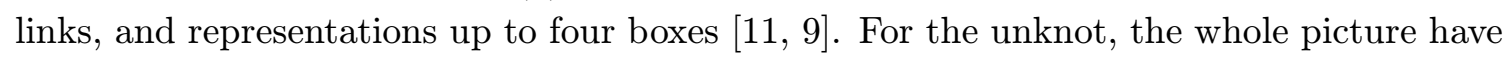
been verified in complete detail $\left[\begin{array}{l}18, \\ 1\end{array} 1 \overline{2} 2\right]$. The form of $F(V)$ for the unknot can be easily computed in Chern-Simons gauge theory,

$$
F(V)=\sum_{d=1}^{\infty} \frac{\lambda^{\frac{d}{2}}-\lambda^{-\frac{d}{2}}}{2 d \sin \left(\frac{d x}{2}\right)} \operatorname{Tr}_{\square} V^{d},
$$

leading to an expansion ( $(2 i)$ ) of the form:

$$
F_{g,(0, \cdots, 0,1,0, \cdots, 0)}(\lambda)=\frac{\left(1-2^{1-2 g}\right)\left|B_{2 g}\right|}{(2 g) !} d^{2 g-2}\left(\lambda^{\frac{d}{2}}-\lambda^{-\frac{d}{2}}\right),
$$

where the 1 in $F_{g,(0, \cdots, 0,1,0, \cdots, 0)}$ is located in the $d^{\text {th }}$ position. Form these equations one can easily read the numbers which correspond to the open-string analogs of Gromov-Witten invariants, $N_{g, \vec{k}}^{\mathcal{Q}}$, in $\left(\underline{B_{i}}\right)$, as well as the new integer invariants present in the general expression (iii): $N_{\square, 0, \frac{1}{2}}=-N_{\square, 0,-\frac{1}{2}}=1$.

Many issues remain open in this context and much work is being carried out to unravel the consequences of this new connection between theoretical physics and topology. I hope 
that in this short note I have convinced the reader of the emergence of a fascinating interplay between string theory, knot theory and enumerative geometry which opens new fields of study.

I would like to thank the organizers of the International Europhysics Conference on High Energy Physics for inviting me to deliver a talk. I would like to thank also M. Mariño for collaborations and discussions on many of the topics described here. This work is supported in part by Ministerio de Ciencia y Tecnología under grant PB96-0960, and by Xunta de Galicia under grant PGIDT00-PXI-20609.

\section{References}

[1] E. Witten, "Quantum field theory and the Jones polynomial", Commun. Math. Phys. 121 (1989) 351.

[2] V.F.R. Jones, "Hecke algebras representations of braid groups and link polynomials", Ann. of Math. 126 (1987) 335; V.G. Turaev, "The Yang-Baxter equation and invariants of links", Inv. Math. 92 (1988) 527.

[3] J.M.F. Labastida, "Chern-Simons Gauge Theory: Ten Years After", hep-th/9905057, Trends in Theoretical Physics II, H. Falomir, R. Gamboa, F. Schaposnik, eds. AIP, New York, 1999, CP 484, 1.

[4] E. Witten, "Chern-Simons gauge theory as a string theory", hep-th/9207094, in The Floer memorial volume, H. Hofer, C.H. Taubes, A. Weinstein and E. Zehner, eds., Birkhäuser 1995, p. 637.

[5] O. Aharony, S. Gubser, J. Maldacena, H. Ooguri and Y. Oz, "Large $N$ field theories, string theory and gravity", hep-th/9905111, Phys. Rep. 323 (2000) 183.

[6] R. Gopakumar and C. Vafa, "On the gauge theory/geometry correspondence", hep-th/9811131, Adv. Theor. Math. Phys. 3 (1999) 1415.

[7] C. Faber and R. Pnadharipande, "Hodge integrals and Gromov-Witten theory", math.AG/9810173.

[8] H. Ooguri and C. Vafa, "Knot invariants and topological strings", hep-th/9912123, Nucl. Phys. B577 (2000) 419.

[9] J.M.F. Labastida, M. Mariño and C. Vafa, "Knots, links and branes at large $N$ ", hep-th/0010102, J. of High Energy Physics 0011 (2000) 007.

[10] J.M.F. Labastida, Marcos Mariño, "A new point of view in the theory of knot and link invariants", math.qa/0104180.

[11] J.M.F. Labastida and M. Mariño, "Polynomial invariants for torus knots and topological strings", hep-th/0004196, Commun. Math. Phys. 217 (2001) 423; P. Ramadevi and T.

Sarkar, "On link invariants and topological string amplitudes," hep-th/0009188, Nucl. Phys. B600 (2001) 487.

[12] S. Katz and M. Liu, "Enumerative geometry of stable maps with Lagrangian boundary conditions and multiple covers of the disc," math.AG/0103074; J. Li and Y.S. Song, "Open string instantons and relative stable morphisms," hep-th/0103100. 